\title{
Epigenetic silencing of olfactomedin-4 enhances gastric cancer cell invasion via activation of focal adhesion kinase signaling
}

\author{
Li-Li Guo ${ }^{1}$, Zhao-Cai He ${ }^{2, *}$, Chang-Qing Yang ${ }^{1}$, Pei-Tang Qiao ${ }^{1}$ \& Guo-Ling Yin ${ }^{3}$ \\ ${ }^{1}$ Department of Gastroenterology, Heping Hospital, Changzhi Medical College, ${ }^{2}$ Department of General Surgery, Heping Hospital, \\ Changzhi Medical College, ${ }^{3}$ Department of radiotherapy, Heping Hospital, Changzhi Medical College, Changzhi, Shanxi 046000, China
}

\begin{abstract}
Downregulation of olfactomedin-4 (OLFM4) is associated with tumor progression, lymph node invasion and metastases. However, whether or not downregulation of OLFM4 is associated with epigenetic silencing remains unknown. In this study, we investigate the role of OLFM4 in gastric cancer cell invasion. We confirm the previous result that OLFM4 expression is increased in gastric cancer tissues and decreases with an increasing number of metastatic lymph nodes, which are associated with OLFM4 promoter hypermethylation. Overexpression of OLFM4 in gastric cancer cells had an inhibitory effect on cell invasion. Furthermore, we found that focal adhesion kinase (FAK) was negatively correlated with OLFM4 in regards to lymph node metastasis in gastric cancer tissues. Also, inhibition of FAK induced by OLFM4 knockdown resulted in a decrease in cell invasion. Thus, our study demonstrates that epigenetic silencing of OLFM4 enhances gastric cancer cell invasion via activation of FAK signaling. [BMB Reports 2015; 48(11): 630-635]
\end{abstract}

\section{INTRODUCTION}

Olfactomedin-4 (OLFM4), a glycoprotein belonging to the olfactomedin family, which comprises at least 13 members, is characterized by a C-terminal olfactomedin domain (1). OLFM4 gene expression is regulated by the transcription factor NF-kappa $\mathrm{B}$ and enhanced by granulocyte colony-stimulating factor (G-CSF) via activation of phosphatidylinositol-3-kinase (PI3K) (2). OLFM4 is able to interact with cell surface proteins. Extracellular OLFM4 can serve as a bridge between the lectins of neighboring cells and participate in cell adhesion (3). OLFM4 can also interact with cell adhesion glycoproteins, such as cadherins (4); its overexpression results in decreased

*Corresponding author. Tel: +13935599013; Fax: +0731-3039231; E-mail: 13935599013@163.com

http://dx.doi.org/10.5483/BMBRep.2015.48.11.130

Received 28 June 2015, Revised 20 July 2015, Accepted 20 August 2015

Keywords: Focal adhesion kinase, Gastric cancer, Invasion, Matrix metalloproteinases, Metastasis, Olfactomedin-4 cell adhesion and migration in HT-29 colorectal cancer cells (5), suggesting that one of the functions of OLFM4 is to regulate cell adhesion and migration.

OLFM4 has been implicated in breast cancer, lung cancer, prostate cancer and digestive tumors (6). Gastric cancer is the second leading cause of cancer-related death in the world (7). Upregulation of OLFM4 at the mRNA and protein levels is observed in gastric and colorectal cancers. Furthermore, it is increased in the early stages of tumor development, though it is much reduced in the more advanced stages of tumor development (8). Downregulation of OLFM4 is associated with tumor progression, lymph node invasion and metastases (9). These studies suggest that OLFM4 plays a role in the early stages of tumor initiation and the advanced stages of tumor invasion.

A wide range of genetic and epigenetic abnormalities, including mutations and hypermethylation, are responsible for the development and progression of gastric carcinoma (10). Epigenetic silencing of some key genes, such as E-cadherin, appears to be the critical carcinogenic event (11). However, whether or not downregulation of OLFM4 is associated with epigenetic silencing remains unknown.

In this study, we aim to investigate whether the epigenetic silencing of OLFM4 in gastric cancer plays a role in cell invasion. Our results demonstrate that epigenetic silencing of OLFM4 enhances gastric cancer cell invasion via activating focal adhesion kinase (FAK) signaling.

\section{RESULTS}

The expression of OLFM4 is associated with its promoter methylation

To investigate the role of OLFM4 in gastric cancer, we made 70 samples of gastric cancer tissues and 10 samples of adjacent tissues into microarrays. The results of immunostaining showed that the expression of OLFM4 was significantly increased in gastric cancer tissues compared to adjacent tissues, but was reduced with increasing number of metastatic lymph nodes (Fig. 1A). In contrast, we found that the expression of FAK was significantly higher in gastric cancer tissues than in adjacent tissues. We also observed that the expression of FAK was increased with increasing number of metastatic lymph nodes (Fig. 1B). In in vitro experiments, we first investigated the 

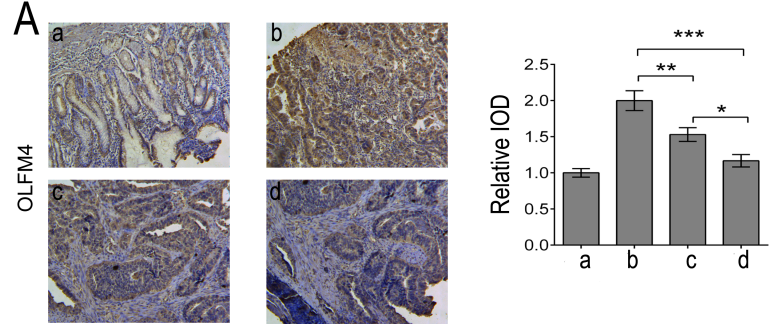

B
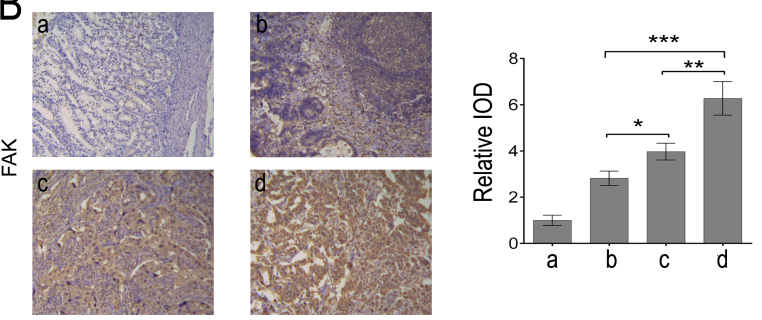

Fig. 1. The expression of OLFM4 and FAK in gastric cancer tissues. (A) Representative images (left) of immunostaining with OLFM4 in adjacent (a) and gastric cancer with 0 lymph node metastases (b), 1-2 lymph node metastases (c) and 3-4 lymph node metastases (d). Quantification of the integral optical density (IOD) for OLFM4 immunostaining (right). (B) Representative images (left) of immunostaining with FAK in adjacent (a) and gastric cancer with 0 lymph node metastases (b), 1-2 lymph node metastases (c) and 3-4 lymph node metastases (d). Quantification of IOD for FAK immunostaining (right). Data are expressed as mean \pm standard deviation, one way analysis of variance (ANOVA) with Bonferroni t post-test was used to analyze the data. ${ }^{*} \mathrm{P}<0.05, * * \mathrm{P}<0.01, * * * \mathrm{P}<0.001$.

invasive ability of 3 gastric cell lines, HGC27, SGC7901, and MGC803. The most invasive were the MGC803 cells, and the least was HGC27 (Fig. 2A). Secondly, we found that the expression level of OLFM4 was higher in gastric cancer cells than in the human gastric epithelial cell line GES-1; its expression was reduced with increasing invasive ability in gastric cancer cells (Fig. 2B). Furthermore, to investigate whether the silencing of OLFM4 in the more advanced cancer cell lines was caused by promoter methylation, we extracted the genomic DNA from gastric cell lines HGC27, SGC7901 and MGC803 to perform bisulfite genomic sequencing PCR. We found that the promoter of OLFM4 was hypermethylated in gastric cancer cells, and the methylation was higher in SGC7901 and MGC803 cells than in HGC27 cells, especially at sites $-706,-691$, -22 and +9 (Fig. 2C). These results indicate that the downregulation of OLFM4 in gastric cancer cells with highly invasive ability is associated with promoter hypermethylation.

\section{Upregulation of OLFM4 inhibits cell invasion in gastric cancer cells}

We further investigated the role of OLFM4 on cell invasion in gastric cancer cells. We downregulated the expression of OLFM4 by transfecting OLFM4-sh lentivirus into SGC7901 and MGC803 cells, and induced the expression of OLFM4 by transfecting OLFM4 lentivirus or treating with $15 \mathrm{nM}$ 5-Aza (a well-known demethylation agent). We found that compared to a negative control (NC) group, downregulation of OLFM4 significantly increased cell invasion, whereas upregulation of OLFM4 dramatically reduced cell invasion. Importantly, 5-Aza treatment also inhibited cell invasion (Fig. 3A, B). These results suggest that OLFM4 represses gastric cancer cell invasion, and this repression is associated with promoter methylation. Moreover, to explore the invasion-related downstream molecules regulated by OLFM4, we analyzed the expression of p-FAK, FAK, MMP2 and MMP9. As shown in Fig. 3C and 3D, we found that downregulation of OLFM4 by shRNA significantly increased the expression of p-FAK, MMP2 and MMP9. Also, 5-Aza treatment strongly enhanced the expression of OLFM4, which resulted in a decrease in the expression of p-FAK, MMP2 and MMP9. Thus, we propose that the inhibitory effect of OLFM4 on gastric cancer cell invasion may be regulated by FAK signaling.

\section{Inhibition of FAK expression reverses the effect of OLFM4-sh on gastric cancer cell invasion}

To investigate whether the effect of OLFM4 on cell invasion involved FAK signaling, we treated SGC7901 and MGC803 cells with OLFM4-sh lentivirus or FAK inhibitor PF-00562271 (PF) alone, or both together. Our results indicate that downregulation of OLFM4 by shRNA significantly increased the expression of $\mathrm{p}-\mathrm{FAK}, \mathrm{MMP2}$ and MMP9. PF treatment had the opposite result. Co-treatment with OLFM4-sh and PF partially neutralized the inhibitory effects of PF on MMP2 and MMP9 expression (Fig. 4A). In line with our results mentioned above, as shown in Fig. 4B, downregulation of OLFM4 resulted in a significant enhancement of cell invasion. FAK inhibitor (PF) significantly inhibited the invasive ability of SGC7901 and MGC803 cells. Importantly, FAK inhibitor treatment mostly blocked the enhanced role of OLFM4-sh on cell invasion. These results demonstrate that the inhibitory effect of OLFM4 on gastric cancer cell invasion was regulated by FAK signaling.

\section{DISCUSSION}

In this study, we found that the expression of OLFM4 and FAK was increased in gastric cancer tissues, whereas the expression of OLFM4 was reduced with an increasing number of metastatic lymph nodes. Previous studies have shown that OLFM4 is increased in several types of cancer, including breast cancer, lung cancer, colorectal cancer and gastric cancer (12). In addition, OLFM4 expression is dependent on the status of differentiation of cancer cells. It was found that OLFM4 expression is increased in well- or moderately-differentiated gastric tissues, whereas its level is much lower in poorly differentiated or undifferentiated tumors (13). Downregulation of OLFM4 is associated with tumor progression, lymph node invasion and metastases in gastric cancer (9). Oue $\mathrm{N}$ et al. found that 
A

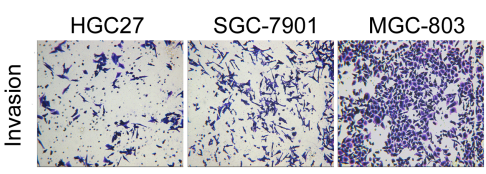

C

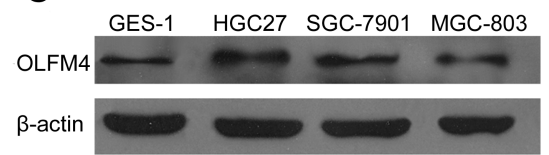

E 5'-GAGCG(-706)GGCACTGGGTCATCG(-691)GGA TCC....... CAAAACG(-587) TTGCTG.......CAACG(-511) ACAACTTGGGCAATAGGAACTGAAGAGGGAAGCT CAGTCG(-471)CTCCTGG.......CAGTTCACACG(-116) TTCCCT.......AGAGCCAGCG(-22)GCTCCAGCTAAG AGGACAAG ATG AGGCC.

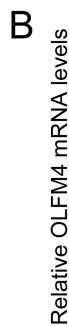

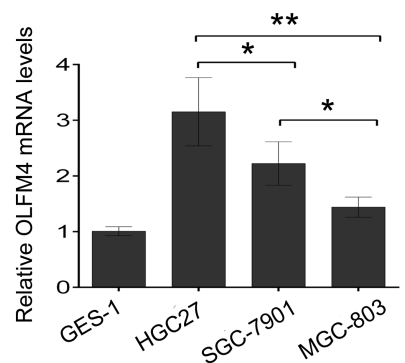

D

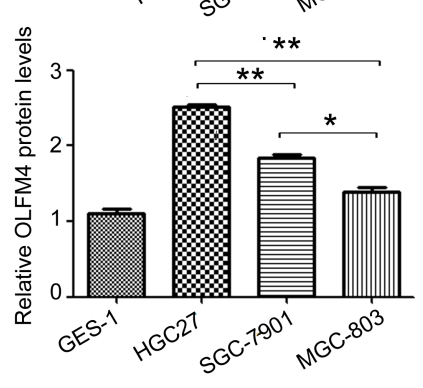

$\mathrm{F}$

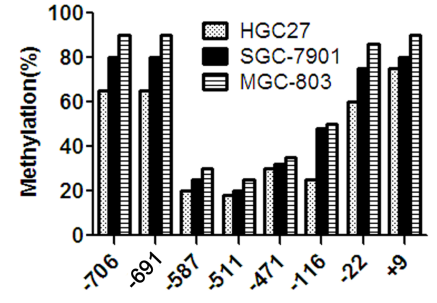

Fig. 2. Hypermethylation of OLFM4 in gastric cancer cell lines. (A) The invasion ability of HGC-27, SGC-7901 and MGC-803 cells measured by transwell assay. (B) OLFM4 mRNA levels were significantly decreased with increasing invasive ability of gastric cancer cells. (C, D) OLFM4 protein levels were significantly decreased with increasing invasive ability of gastric cancer cells. (E, F) The OLFM4 promoter was hypermethylated especially at the sites -706 , $-691,-22$ and +9 . Data are expressed as mean \pm standard deviation; one way analysis of variance (ANOVA) with Bonferroni $t$ post-test was used to analyze the data. ${ }^{*} \mathrm{P}<0.05,{ }^{*} * \mathrm{P}<0.01$.

A

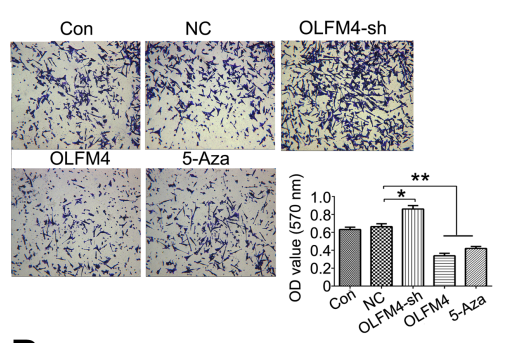

B

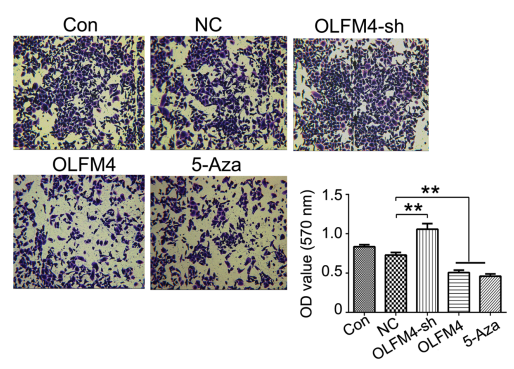

C

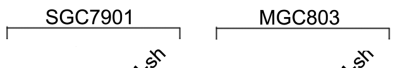

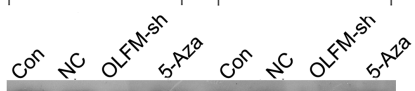
OLFM4 $--\infty-\cdots$ p-FAK(Tyr397) - - - - - -
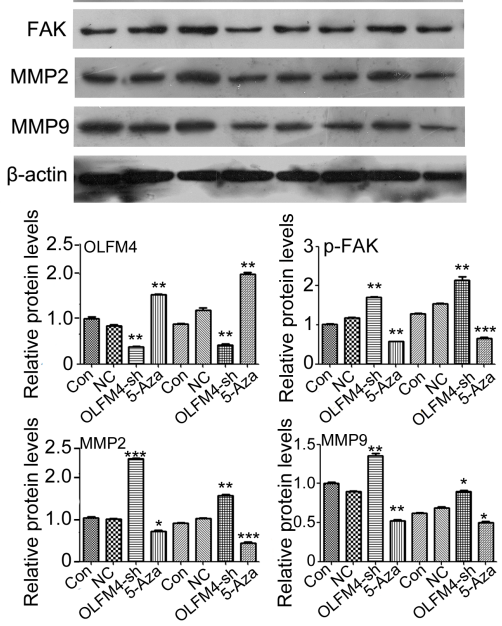

Fig. 3. OLFM4 reduces invasive ability of gastric cancer cells, and regulates the expression of p-FAK, MMP2 and MMP9. SGC-7901 (A) and MGC-803 (B) cells were transfected with OLFM4-sh, OLFM4 lentivirus, or 5-Aza for $48 \mathrm{~h}$. Invasive ability was measured by transwell assay. (C) SGC-7901 and MGC-803 cells were transfected with OLFM4-sh, OLFM4 lentivirus, or treated with 5-Aza for $48 \mathrm{~h}$. Expression levels of OLFM4, p-FAK, FAK, MMP2 and MMP9 protein were measured by western blot (upper); quantification of the bands is shown (lower). 5-Aza treatment induced OLFM4 expression and reduced p-FAK MMP2 and MMP9 expression compared to NC. Downregulation of OLFM4 by OLFM4-sh led to an increase in p-FAK, MMP2 and MMP9 expression compared to NC. Data are expressed as mean \pm standard deviation. One way analysis of variance (ANOVA) with Bonferroni t posttest was used to analyze the data. $* \mathrm{P}<$ $0.05, * * \mathrm{P}<0.01$. p-FAK, phosphorylatedFAK; Con, control, the cells are untreated; $\mathrm{NC}$, negative control, the cells are treated with empty lentivirus. 


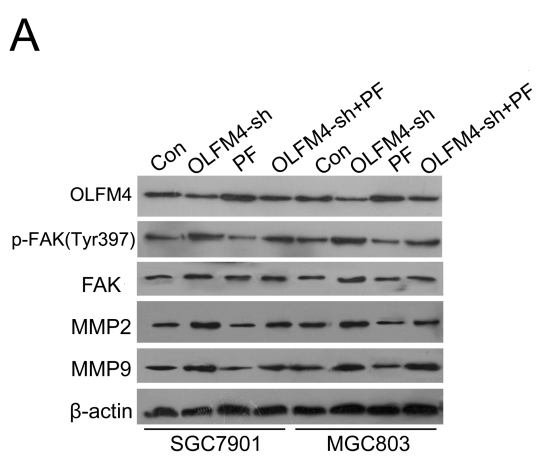

B

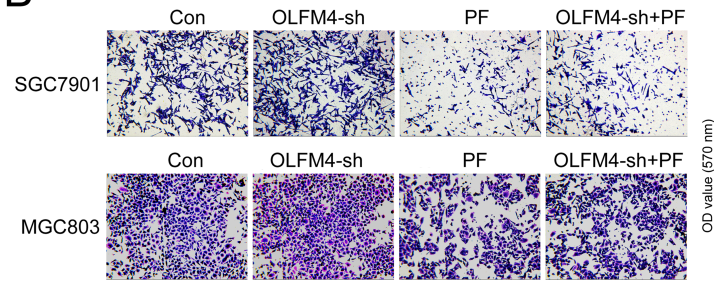

$\stackrel{\frac{\infty}{\Phi}}{\stackrel{\frac{\omega}{\omega}}{1} 1.5}$ OLFM4

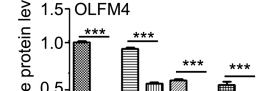

$\sum_{\frac{\pi}{0}}^{\frac{\pi}{0}}$

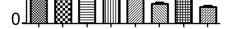

OLM4-sh - + + + + + OLFM4-sh - + + + + +
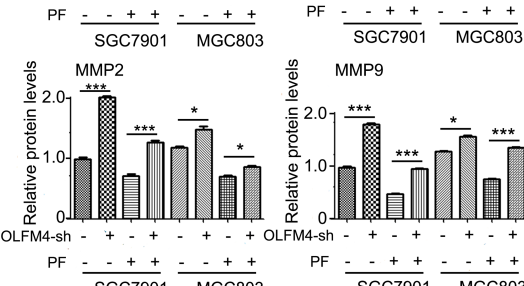

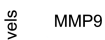

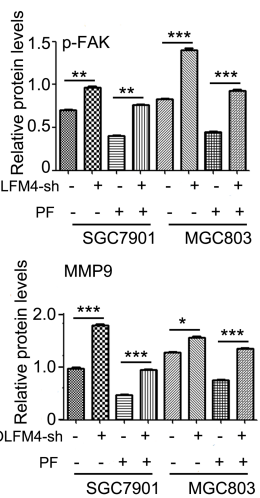

Fig. 4. Inhibition of FAK reduces the invasive ability that is enhanced by OLFM4 downregulation. SGC-7901 and MGC-803 cells were treated with OLFM4-sh lentivirus or FAK inhibitor (PF) alone, or co-treated with OLFM4-sh lentivirus and PF. Western blot was used to detect the expression of OLFM4, p-FAK, FAK, MMP2 and MMP9 (left); quantification of the bands is shown (right). An unpaired two tailed student's t-test was used analyze the data. ${ }^{*} \mathrm{P}<0.05,{ }^{* *} \mathrm{P}<0.01$ alyze the data.
$* * * \mathrm{P}<0.001$. Cellular invasive ability was measured by transwell assay after indicated treatment. Data are expressed as mean \pm standard deviation from three independent experiments. One was analysis of variance (ANOVA) with Bonferroni T post-test was
used to analysis the data. ${ }^{*} \mathrm{P}<0.05,{ }^{* * *} \mathrm{P}$ $<$ 0.001; \# $\mathrm{P}<0.01$, \#\# $\mathrm{P}<0.001 \mathrm{VS}$ OLFM4-sh group.

OLFM4 expression in gastric cancer tissues and the serum of gastric cancer patients is higher in stage $\mathrm{I} / \mathrm{II}$ cases than in stage III/IV cases (14). It is possible that the expression of OLFM4 in the early stages of gastric cancer is compensatorily upregulated. On the other hand, given the inhibitory effects of OLFM4 on cancer cell invasion, its expression is suppressed during the late stages of gastric cancer (cancer cell invasion and metastases). Downregulation of OLFM4 in an advanced tumor is associated with a decrease in patient survival (15), suggesting that OLFM4 not only plays a role in the early stages of tumor initiation, but also has an inhibitory role on cell invasion and metastases in advanced stages of tumor development.

Furthermore, our in vitro experiments revealed that OLFM4 expression is reduced with increasing cell invasive ability, and this effect is silenced by promoter hypermethylation. DNA methylation alterations are induced by carcinogenetic factors at both the early and advanced stages in various organs (16). It has been suggested that DNA methylation profiles may determine tumor aggressiveness and patient outcomes (17). Accumulative studies have shown that key players in gastric cancer are regulated by changes in DNA methylation patterns at their promoter $\mathrm{CpG}$ islands (18). Hypermethylation of $\mathrm{CpG}$ islands results in the silencing of neighboring genes, and promoters of tumor-suppressor genes, such as P53 and runt-related transcription factor 3 , are often methylated in tissue taken from gastric cancer patients (19). In the present study, downregulation of OLFM4 that is silenced by a hypermethylated promoter in advanced gastric cancer is associated with invasive behavior.
FAK is an important intracellular signaling molecule that mediates crosslinking of intracellular signaling networks and plays a key role in cell migration and invasion (20). Previous studies showed that FAK expression is increased in gastric cancer tissue, especially in poorly differentiated gastric cancer tissue (9). Interestingly, we now find that the expression of FAK has a negative relationship with OLFM4 in gastric cancer tissue. What's more, we found that enhancement of cell invasion by OLFM4-sh was diminished by a FAK inhibitor, which led to a decrease in the expression of matrix metalloproteinase 2 (MMP2) and MMP9. PF is a potent ATP-competitive inhibitor of FAK. PF may inhibit phosphorylation of FAK both in vitro and in vivo, and subsequently reduce protein levels of MMP2 and MMP9 (21). FAK signaling also plays a critical role in the production of matrix metalloproteinases including MMP2 and MMP9 and subsequently activates tumor invasion (22). Overexpressed OLFM4 in HT-29 colorectal cancer cells led to a visible change in cell morphology with the cells losing contact with one another and becoming rounded, resulting in decreased cell adhesion and migration; this effect may be mediated by interactions with adhesion molecules, the cytoskeleton and the extracellular matrix (23). Park KS et al. found that overexpression of OLFM4 reduced expression of MMP9, and forced expression of MMP9 attenuated the inhibitory activity of OLFM4 on migration and invasiveness in mouse melanoma cells (24). In this study, we reveal that OLFM4 expression is negatively correlated with the activity of FAK signaling, and downregulation of OLFM4 by shRNA enhances the invasive ability of gastric cancer cells, which is attenuated by a FAK inhibitor. These results suggest that OLFM4 
suppresses gastric cancer cell invasion partly via activation of FAK signaling.

In conclusion, our findings provide experimental evidence that OLFM4 functions as an invasive suppressor especially in advanced gastric cancer via regulating FAK/MMP2/MMP9 signaling. However, other studies suggest that depletion of OLFM4 significantly inhibits tumorigenicity of gastric cancer SGC-7901 and MGC803 cells and sensitizes gastric cancer cells to hydrogen peroxide or tumor necrosis factor alpha (25). Thus, balancing and stabilizing OLFM4 expression levels in gastric cancer is an important area for future exploration.

\section{MATERIALS AND METHODS}

\section{Gastric cancer tissue samples}

A total of 80 tissue samples, including 10 adjacent tumor tissues, 15 early gastric carcinoma tissues, and 55 advanced gastric cancer tissues were purchased from AURAGEN Inco. (TC0130, Changsha, China). Informed written consent was given by all subjects. All samples were collected and identified by histopathological evaluation, and stored at $-80^{\circ} \mathrm{C}$ until used.

\section{Cell culture}

Human gastric cancer cell lines HGC27, SGC-7901, and MGC-803, and human gastric epithelial cells GES-1, were obtained from American Type Culture Collection (ATCC, Manassas, VA, USA). All cells were cultured in RPMI1640 medium (Invitrogen Life Technologies, Carlsbad, CA, USA), supplemented with $10 \%(\mathrm{v} / \mathrm{v})$ fetal bovine serum (FBS) (Invitrogen Life Technologies) at $37^{\circ} \mathrm{C}$ in a humidified $5 \% \mathrm{CO}_{2}$ incubator.

\section{Cell treatment}

Lentivirus overexpressed and knockdown OLFM4 were designed and purchased from Genechem (Genechem, Shanghai, China). Following $48 \mathrm{~h}$ transfection, the expression of OLFM4 was detected by western blotting, and the transfected cells were used for further analysis. Cells transfected with empty lentivirus were used as a negative control. Cells were treated with $15 \mathrm{nM}$ 5'-Aza-deoxycytidine (5-Aza, Sigma-Aldrich, St. Louis, MO, USA) or 1.5 nM PF-00562271, a FAK inhibitor (Selleck, Houston, TX, USA) for $48 \mathrm{~h}$ and then used for further analysis.

\section{Immunohistochemical (IHC) staining}

All tissue samples were divided into three slices of tissue microarray as previously described, with each sample in duplicate or triplicate. Slides were deparaffinized in dimethylbenzene and dehydrated in an alcohol gradient. Slides were immersed in $3 \%$ hydrogen peroxide to inactivate endogenous peroxidase, and then retrieved in citric acid buffer $(\mathrm{pH} 6.0)$ in a microwave for $15 \mathrm{~min}$. After cooling, the slices were rinsed with TBST,blocked with normal goat serum, and incubated with primary antibody rabbit monoclonal anti-OLFM4 (1:500 dilution, cell signaling technology) or rabbit monoclonal an-
ti-FAK antibody (1:500 dilution; Cell Signaling Technology, Inc., Danvers, MA, USA) overnight at $4^{\circ} \mathrm{C}$. The slides were then washed with TBST and incubated with anti-rabbit secondary antibody (Boster, Wuhan, China) for $2 \mathrm{~h}$ at $37^{\circ} \mathrm{C}$. The sections were then washed with TBST and stained with a DAB Detection Kit (Solarbio, Beijing, China). Finally, the sections were counterstained with hematoxylin.

\section{Westem blot analysis}

RIPA lysis buffer (Boster, Wuhan, China) was used to extract protein from indicated cells. BCA Protein Assay Kit (Thermo Scientific, USA) was used to measure protein concentration. A total of $60 \mu \mathrm{g}$ of protein was separated on 10\% SDS-PAGE gels and blotted onto $0.22 \mu \mathrm{m}$ nitrocellulose membranes (Boster). The membranes were blocked for 2 hours with $5 \%$ nonfat dry milk diluted with tris-buffered saline (TBS) and incubated with primary antibodies (rabbit monoclonal anti-OLFM4 $(1: 2,000)$, rabbit monoclonal anti-phosphorylated-FAK antibody (1:500), rabbit monoclonal anti-FAK antibody $(1: 1,000)$, rabbit monoclonal anti-MMP2 $(1: 1,000)$, rabbit monoclonal anti-MMP9 $(1: 3,000)$, and mouse monoclonal anti- $\beta$-actin $(1: 3,000)$ (Cell Signaling Technology, Inc., Danvers, MA, USA) overnight at $4^{\circ} \mathrm{C}$. The membranes were washed with tris-buffered saline containing $0.1 \%$ Tween 20 (TBST), and then incubated with the appropriate horseradish peroxidase-conjugated secondary antibody (goat anti-rabbit, 1:3,000; goat anti-mouse, 1:2,000; Wuhan Boster) for $1 \mathrm{~h}$ at $37^{\circ} \mathrm{C}$. Enhanced chemiluminescence reagent (Merck Millipore, Germany) was used to detect the signal on the membrane. The data was analyzed via densitometry using Image-Pro plus 6.0 software (Media Cybernetics, Rockville, MD, USA) and normalized to the expression of the internal control ( $\beta$-actin).

\section{Measurement of OLFM4 promoter CpG island methylation status by bisulfite genomic sequencing PCR (BSP)}

Genomic DNA was extracted from HGC27, SGC-7901 and MGC-803 cells with the TaKaRa Genomic DNA Extraction Kit (TaKaRa Co., China). Genomic DNA (1 $\mu$ g per sample) was modified with bisulfite using the Epitect Bisulfite Kit Protocol (Qiagen) following the manufacturer's instructions, and the modified DNA was amplified using the following primers: OLFM4 forward, AAAGGTGTGTGAAATGTTGAG, and reverse, CTCTCCCCCATTTTACT. The PCR products were gel extracted (Qiagen) to confirm that a single band had been obtained and were then sequenced by Invitrogen.

\section{Transwell assay}

The indicated cells were starved for $24 \mathrm{~h}$ and then resuspended in serum-free medium and added to the upper chamber of the tissue culture plate (BD, Frankin Lakes, New Jersey, United States). The lower chamber was filled with medium containing $10 \%$ FBS. Following $48 \mathrm{~h}$ incubation, cells attached to the bottom were fixed and stained with crystal violet for $45 \mathrm{~min}$ and air dried. The optical density (OD) at $570 \mathrm{~nm}$ 
of crystal violet dissolved by $10 \%$ acetic acid was detected by an enzyme immunoassay analyzer (Synergy ${ }^{\mathrm{TM}} \mathrm{Mx}$; BioTek, Winooski, VT, USA).

\section{Statistical analysis}

Statistical analyses were performed using GraphPad Prism 5 software (Graphpad Software, Inc., La Jolla, CA, USA) and the data are presented as the mean \pm standard deviation. An unpaired two-tailed Student's t-test or one way analysis of variance (ANOVA) with Bonferroni t post-test was used to analyze the data depending on conditions. $\mathrm{P}<0.05$ was considered to indicate a statistically significant difference.

\section{REFERENCES}

1. Clemmensen SN, Bohr CT, Rorvig S et al (2012) Olfactomedin 4 defines a subset of human neutrophils. J Leukoc Biol 91, 495-500

2. Chin KL, Aerbajinai W, Zhu J et al (2008) The regulation of OLFM4 expression in myeloid precursor cells relies on NF-kappaB transcription factor. Br J Haematol 143, 421432

3. Sun F, Peatman E, Li C et al (2012) Transcriptomic signatures of attachment, NF-kappaB suppression and IFN stimulation in the catfish gill following columnaris bacterial infection. Dev Comp Immunol 38, 169-180

4. Grover PK, Hardingham JE, Cummins AG (2010) Stem cell marker olfactomedin 4: critical appraisal of its characteristics and role in tumorigenesis. Cancer Metastasis Rev 29, 761-775

5. Besson D, Pavageau AH, Valo I et al (2011) A quantitative proteomic approach of the different stages of colorectal cancer establishes OLFM4 as a new nonmetastatic tumor marker. Mol Cell Proteomics 10, M111-M9712

6. Yu L, Wang L, Chen S (2011) Olfactomedin 4, a novel marker for the differentiation and progression of gastrointestinal cancers. Neoplasma 58, 9-13

7. Sampieri CL, Leon-Cordoba K, Remes-Troche JM (2013) Matrix metalloproteinases and their tissue inhibitors in gastric cancer as molecular markers. J Cancer Res Ther 9, 356-363

8. Jang BG, Lee BL, Kim WH (2015) Olfactomedin-related proteins 4 (OLFM4) expression is involved in early gastric carcinogenesis and of prognostic significance in advanced gastric cancer. Virchows Arch 467, 285-294

9. Luo Z, Zhang Q, Zhao Z, Li B, Chen J, Wang Y (2011) OLFM4 is associated with lymph node metastasis and poor prognosis in patients with gastric cancer. J Cancer Res Clin Oncol 137, 1713-1720

10. Yamanoi K, Arai E, Tian Y et al (2015) Epigenetic clustering of gastric carcinomas based on DNA methylation profiles at the precancerous stage: its correlation with tumor aggressiveness and patient outcome. Carcinogenesis 36,
509-520

11. Carneiro P, Figueiredo J, Bordeira-Carrico R et al (2013) Therapeutic targets associated to E-cadherin dysfunction in gastric cancer. Expert Opin Ther Targets 17, 1187-1201

12. Koshida S, Kobayashi D, Moriai R, Tsuji N, Watanabe N (2007) Specific overexpression of OLFM4(GW112/HGC-1) mRNA in colon, breast and lung cancer tissues detected using quantitative analysis. Cancer Sci 98, 315-320

13. Liu W, Zhu J, Cao L, Rodgers GP (2007) Expression of hGC- 1 is correlated with differentiation of gastric carcinoma. Histopathology 51, 157-165

14. Oue N, Sentani K, Noguchi T et al (2009) Serum olfactomedin 4 (GW112, hGC-1) in combination with Reg IV is a highly sensitive biomarker for gastric cancer patients. Int J Cancer 125, 2383-2392

15. Suh YS, Lee HJ, Jung EJ et al (2012) The combined expression of metaplasia biomarkers predicts the prognosis of gastric cancer. Ann Surg Oncol 19, 1240-1249

16. He D, Zhang YW, Zhang NN et al (2015) Aberrant gene promoter methylation of p16, FHIT, CRBP1, WWOX, and DLC-1 in Epstein-Barr virus-associated gastric carcinomas. Med Oncol 32, 92

17. Zouridis H, Deng N, Ivanova T et al (2012) Methylation subtypes and large-scale epigenetic alterations in gastric cancer. Sci Transl Med 4, 140r-156r

18. Dauksa A, Gulbinas A, Endzinas Z, Oldenburg J, El-Maarri O (2014) DNA methylation at selected CpG sites in peripheral blood leukocytes is predictive of gastric cancer. Anticancer Res 34, 5381-5388

19. Zhao C, Bu X (2012) Promoter methylation of tumor-related genes in gastric carcinogenesis. Histol Histopathol $27,1271-1282$

20. Du T, Qu Y, Li J et al (2014) Maternal embryonic leucine zipper kinase enhances gastric cancer progression via the FAK/Paxillin pathway. Mol Cancer 13, 100

21. Roberts WG, Ung E, Whalen P et al (2008) Antitumor activity and pharmacology of a selective focal adhesion kinase inhibitor, PF-562,271. Cancer Res 68, 1935-1944

22. Mon NN, Ito S, Senga T, Hamaguchi M (2006) FAK signaling in neoplastic disorders: a linkage between inflammation and cancer. Ann N Y Acad Sci 1086, 199-212

23. Zhang J, Liu WL, Tang DC et al (2002) Identification and characterization of a novel member of olfactomedin-related protein family, hGC-1, expressed during myeloid lineage development. Gene 283, 83-93

24. Park KS, Kim KK, Piao ZH et al (2012) Olfactomedin 4 suppresses tumor growth and metastasis of mouse melanoma cells through downregulation of integrin and MMP genes. Mol Cells 34, 555-561

25. Liu RH, Yang MH, Xiang $\mathrm{H}$ et al (2012) Depletion of OLFM4 gene inhibits cell growth and increases sensitization to hydrogen peroxide and tumor necrosis factor-alpha induced-apoptosis in gastric cancer cells. J Biomed Sci 19, 38 